\title{
La nécropole romaine de Porta Nocera à Pompéi :
} naissance et développement d'un paysage funéraire romain ( $\mathrm{I}^{\mathrm{er}}$ siècle $\mathrm{av}$. J.-C. $-\mathrm{I}^{\mathrm{er}}$ siècle apr. J.-C.)

La campagne 2015

William Van Andringa, Thomas Creissen et Henri Duday

\section{OpenEdition}

\section{Journals}

Édition électronique

URL : http://journals.openedition.org/cefr/1528

DOI : $10.4000 /$ cefr. 1528

ISSN : 2282-5703

Éditeur

École française de Rome

Référence électronique

William Van Andringa, Thomas Creissen et Henri Duday, «La nécropole romaine de Porta Nocera à Pompéi : naissance et développement d'un paysage funéraire romain (ler siècle av. J.-C. - Ier siècle apr. J.-C.) », Chronique des activités archéologiques de l'École française de Rome [En ligne], Les cités vésuviennes, mis en ligne le 21 mars 2016, consulté le 21 novembre 2019. URL : http:// journals.openedition.org/cefr/1528; DOI : 10.4000/cefr.1528

Ce document a été généré automatiquement le 21 novembre 2019.

(c) École française de Rome 


\section{La nécropole romaine de Porta Nocera à Pompéi : naissance et développement d'un paysage funéraire romain $\left(\mathrm{I}^{\mathrm{er}}\right.$ siècle av. J.-C. - $\mathrm{I}^{\mathrm{er}}$ siècle apr. J.-C.)}

La campagne 2015

William Van Andringa, Thomas Creissen et Henri Duday

\section{NOTE DE L'AUTEUR}

Rédacteur de la chronique : Thomas Creissen et William Van Andringa.

Participants à la campagne 2015 : Marie-Josée Ancel (Archeodunum), Marie Anselmo, Reine-Marie Bérard (École française de Rome), Philippe Brunner, Cécilia Cammas, Marianna Castiglione (Università degli Studi di Pisa), Marie-Caroline Charbonnier, Mathilde Carrive (École française de Rome), Thomas Creissen (Éveha International), Alice Dananai (université de Lille 3), Franck Decanter, Henri Duday (Université de Bordeaux), Johannes Laiho, Pauline Larrieu, Alexia Lattard (université d'Aix-Marseille), Tuija Lind, Adrien Malignas, Florence Monier (CNRS), Pascal Neaud (UMR 7041, ArScan), Clarisse Parra-Prieto, Émilie Portat, Albert Ribera (SIA Ajuntament de Valencia), Federica Maria Riso (Università degli Studi di Modena), Saverio di Rosa, Géraldine Sachau-Carcel, Jean-Baptiste Sinquin (Éveha), William Van Andringa (Helsinki Collegium et Université de Lille 3), Anne-Sophie Vigot (Éveha International), Gilles Warzyniak, Véronique Zech-Matterne (CNRS).

1 Le programme d'étude Porta Nocera 2 a pour ambition d'étudier les modalités de mise en place et de développement d'une nécropole urbaine romaine à partir d'un axe viaire, aménagement essentiel dans l'expression de la mort à l'époque romaine. À ce titre, la 
nécropole de Porta Nocera essentiellement dégagée entre 1952 et 1958 puis en 1983 offre un terrain d'étude privilégié. En effet, les monuments et enclos funéraires qui longent la route de Nocera présentent des structures funéraires (tombes, aires de crémation) bien conservées, jusqu'aux inscriptions en place, qui permettent d'observer les pratiques funéraires en vigueur, cela sur un temps court, 160 ans à peine, puisqu'il nous paraît acquis que les ensembles funéraires bordant la route de Nocera ont été mis en place à partir de la fondation de la colonie romaine en $80 \mathrm{av}$. J.-C. C'est à notre avis la nécessité d'organiser une nécropole qui est à l'origine de l'aménagement du territoire situé au sud-est de la ville, de la naissance d'un paysage nouveau sans doute peu marqué jusque-là sinon par la présence d'une muraille urbaine. La campagne $2015^{1}$ a permis d'ouvrir deux zones, la zone A autour de l'enclos funéraire 26a (nécropole ouest) mis en évidence en 2014 ainsi que la zone B le long de la route de Nocera (nécropole est), à l'emplacement des dégagements de $1983^{2}$ (fig. 1).

Fig. 1 - Vue générale de la voie de Nocera et de la nécropole associée figurant les zones fouillées en 2016.

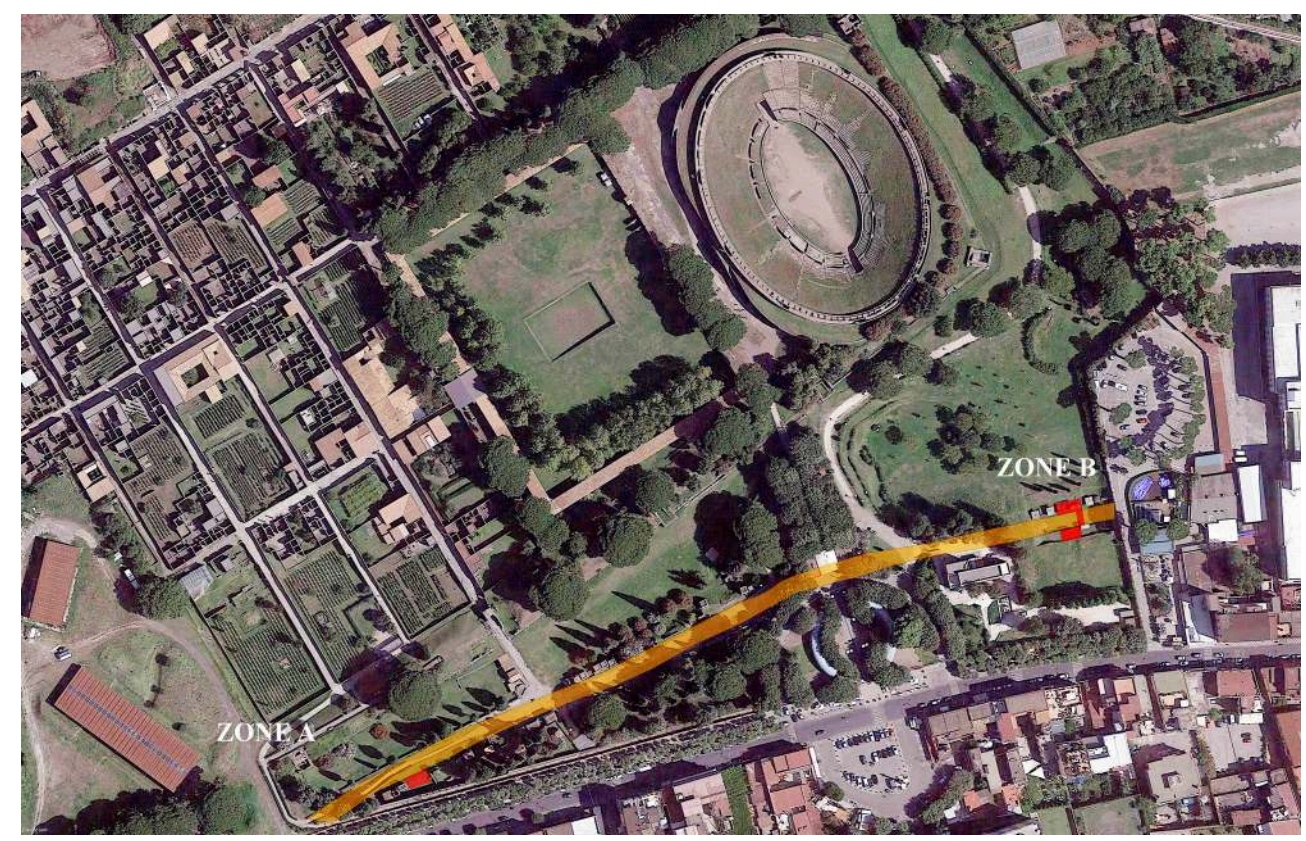

C C GOOgLE EARTH/MISSION ARCHÉOLOgIQUE DE PORTA NOCERA 2.

\section{La Zone A (Porta Nocera Ouest). Près des portes de la ville : fouille de l'ensemble 25c, 26a et 27 OS (voie et espaces funéraires)}

2 Dans la Zone A, la campagne de cette année visait plusieurs objectifs (fig. 2). 
Fig. 2 - Vue générale de la zone A.

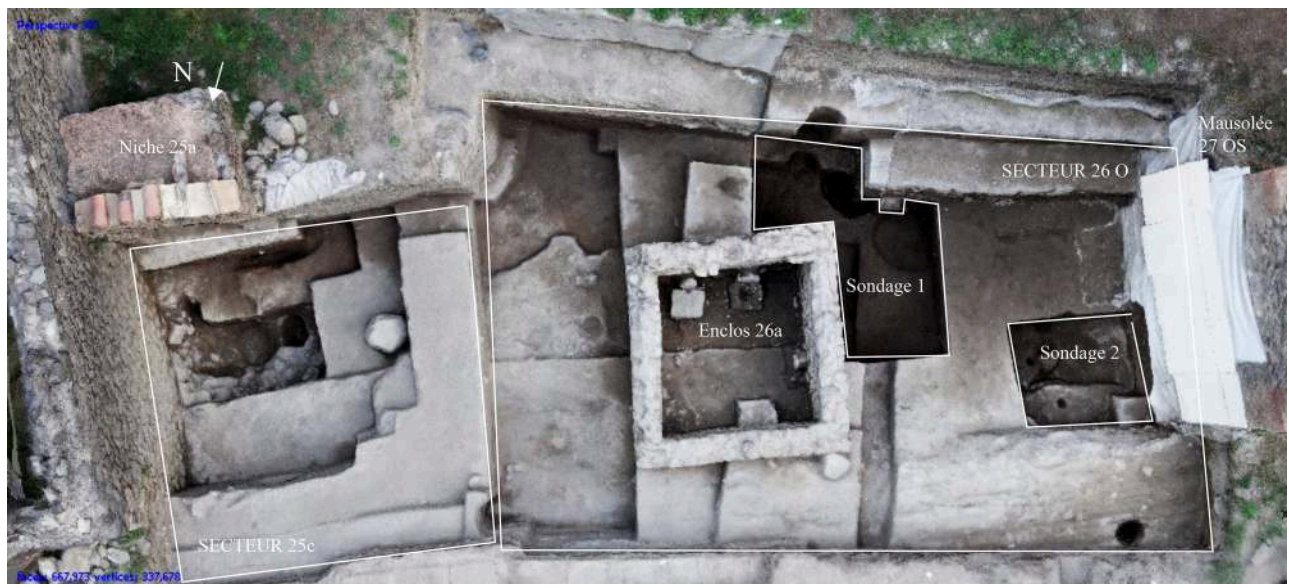

(c) Mission archéologique de Porta Nocera 2.

Il s'agissait notamment d'observer et de comprendre les premières traces d'occupation du secteur, d'établir la chronologie des plus anciennes occupations funéraires, mais aussi de comprendre l'histoire des trois ensembles funéraires identifiés l'an passé $270 S$, 26a et $25 \mathrm{c}-$, et cela depuis leur origine jusqu'à leur désertion. La question des relations entre la voie et les espaces funéraires comme celle des transformations que cet espace de circulation a connues faisaient également partie du programme global (fig. 3).

Fig. 3 - La zone A en cours de fouilles.

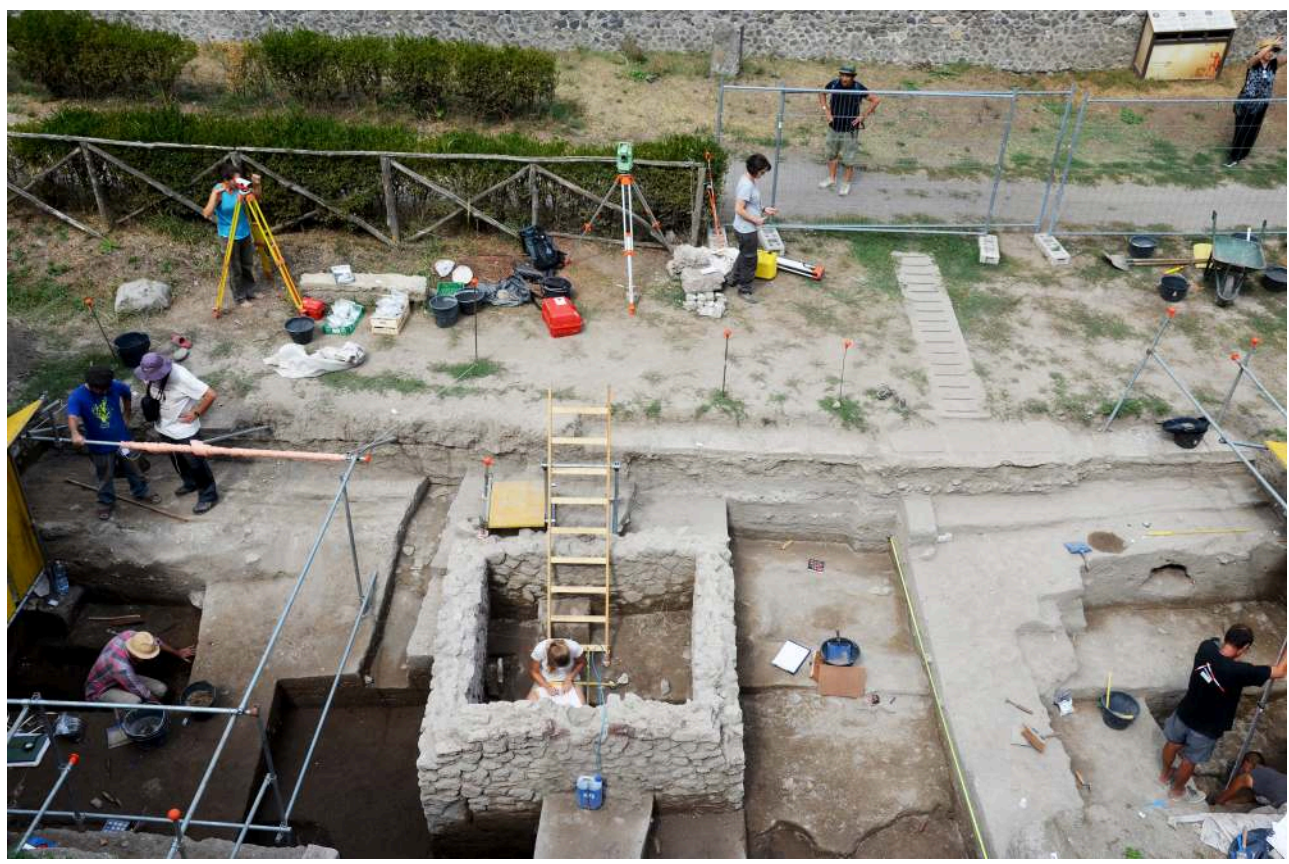

(c) Mission archéologique de Porta Nocera 2.

3 Pour des raisons de temps et de contraintes de sécurité, le substrat n'a pu être atteint qu'en certains endroits. Les données sont toutefois suffisantes pour affirmer que ce secteur faisait office de carrière avant l'installation de la nécropole. Le substrat volcanique mais aussi peut-être des couches de limons y étaient exploités. Il n'est 
malheureusement pas possible de savoir à partir de quand cette exploitation a commencé. Cette carrière bordait vraisemblablement la voie qui devait déjà exister. La roche plonge fortement en direction du sud, et la voie paraît avoir été aménagée sur un replat. Ces informations confirment ce qui avait déjà été observé lors du précédent programme de recherche consacré à cette nécropole (2003-2008).

4 Par la suite, ce secteur se voit conférer un usage funéraire moyennant d'importants travaux de terrassements du fait de la forte irrégularité du terrain. Le plus ancien monument situé dans l'emprise de la zone fouillée est le mausolée 27 OS, qui pourrait remonter au $3^{\mathrm{e}}$ quart du $\mathrm{I}^{\mathrm{er}}$ siècle avant n.è. Aucune sépulture fonctionnant avec ce dernier n'a été identifiée au sein du sondage qu'il a été possible de réaliser sur son flanc est. Ceci signifie que la ou les tombes associé(es) se situe(nt) soit en façade, soit au revers ou bien encore à l'intérieur du monument, autant d'espaces qu'il n'est pas possible d'explorer pour des questions de sécurité. L'étude de ce mausolée devra donc rester incomplète.

Lors de cette campagne, il a pu être démontré que les niveaux de chantier liés à la construction du monument 27 OS ont été recoupés pour l'établissement du monument 26a, situé environ $3 \mathrm{~m}$ plus à l'est : ce dernier est incontestablement plus récent et il est possible que la nécropole se soit progressivement développée à partir de l'ouest. Les fondations de 26a ont pu être atteintes à différents endroits et les niveaux de circulation extérieurs clairement individualisés. Un premier examen du matériel céramique associé invite à envisager une datation augustéenne, mais l'étude est encore en cours.

6 Ce monument, déjà repéré l'an dernier, avait été interprété comme un petit mausolée. Les fouilles de cette année invitent à y reconnaître un enclos à ciel ouvert. Au sud, le mur du fond était surmonté d'un fronton. Seule la corniche inférieure en est conservée. En partie centrale, elle est interrompue par une niche qui abritait une urne funéraire en verre. Lors de la campagne 2014, cette dernière a été retrouvée brisée et en partie vidée de ses ossements. Au commencement de la nouvelle campagne, l'intérieur de cet enclos était encore largement comblé par divers niveaux d'abandons, naturels (colluvions) ou d'origine anthropique (dépotoir hétérogène). Une fois ces derniers démontés, trois tombes, au moins, sont apparues. L'une est marquée par une columelle en lave ; les deux autres sont signalées par des stèles en marbre inscrites. Une épitaphe mentionne la jeune Aphe, morte à six ans; l'autre est celle de Poppaea Cypare, membre de la famille des Poppaei de Pompéi dont serait issue Poppée, l'épouse de Néron.

7 Sous les niveaux d'abandon, le dernier niveau de fréquentation de l'enclos est caractérisé par la présence de plusieurs balsamaires en verre associés aux tombes. 
Fig. 4 - Niveaux de fréquentations à l'intérieur de l'enclos 26a.

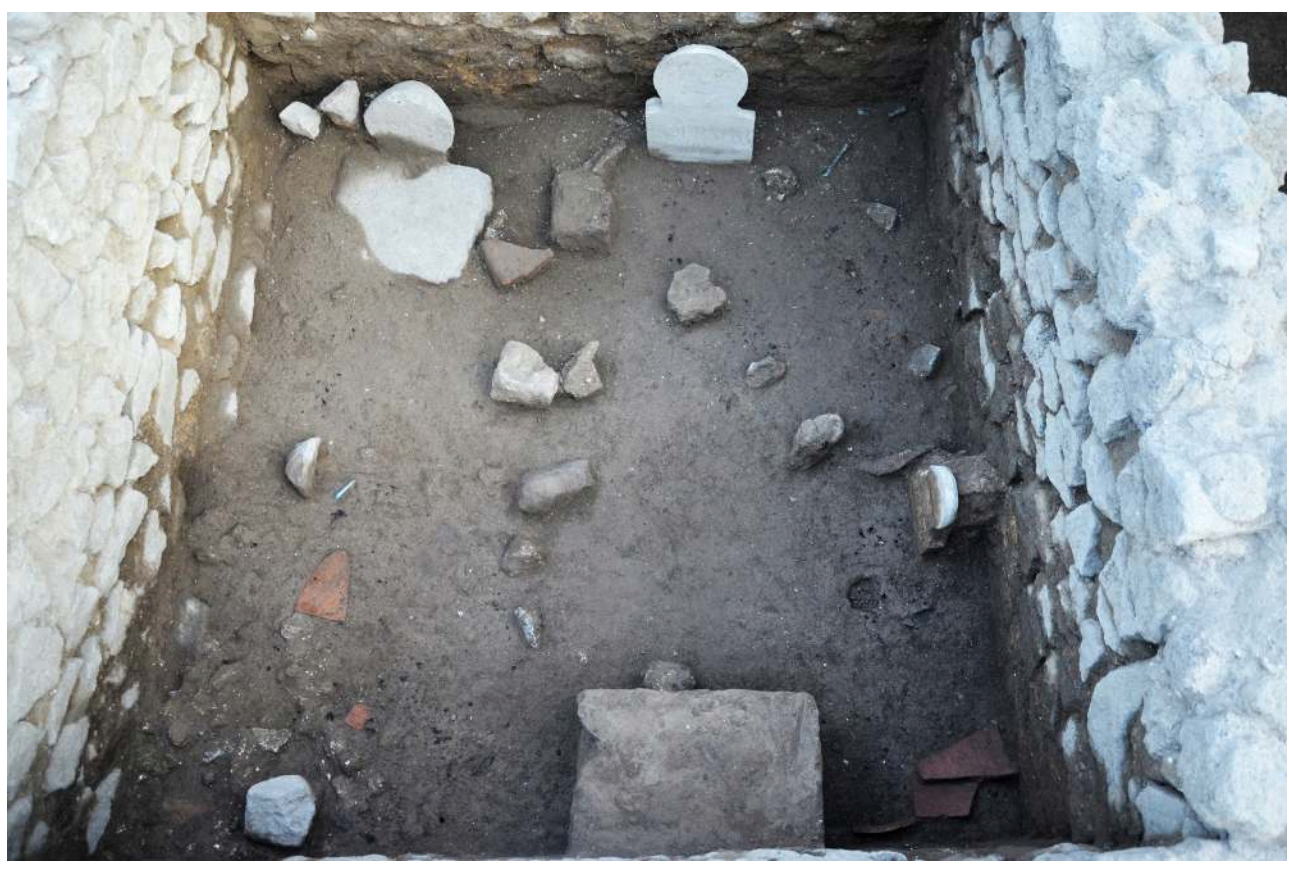

(c) Mission archéologique de Porta Nocera 2

Ces tombes n'ont pas encore été fouillées, mais les dispositifs funéraires apparaissent exceptionnellement bien conservés: plaques de scellement, stèles et conduits à libations sont encore présents et de nombreux éléments jonchent les sols environnants (fig. 4). Pour une des tombes, il est manifeste qu'une partie du tube à libations, au moins, était en matériau périssable. Si l'une des plaques de scellement est en lave, l'autre est constituée de galets enfoncés dans un mortier (?) argileux très meuble, un type d'aménagement jamais rencontré lors des précédentes campagnes engagées sur ce site. La fouille de toutes les tombes de l'enclos est prévue pour l'année 2016.

Le dernier ensemble funéraire est constitué par la concession 25c, qui occupe la partie la plus orientale de la zone fouillée. L'espace situé entre cet ensemble et l'enclos 26a n'a pas été intégralement fouillé et la chronologie relative demande à être affinée. À partir de l'étude du matériel et en se fondant sur les résultats issus du premier programme de recherche consacré à ce secteur de la nécropole, une datation augustéenne semble vraisemblable.

9 Au total, quatre stèles sont présentes dans cette concession, auxquelles correspondent des creusements pour partie aménagés dans le substrat volcanique (fig. 5). 
Fig. 5 - Les tombes anciennes de la concession $25 \mathrm{c}$.

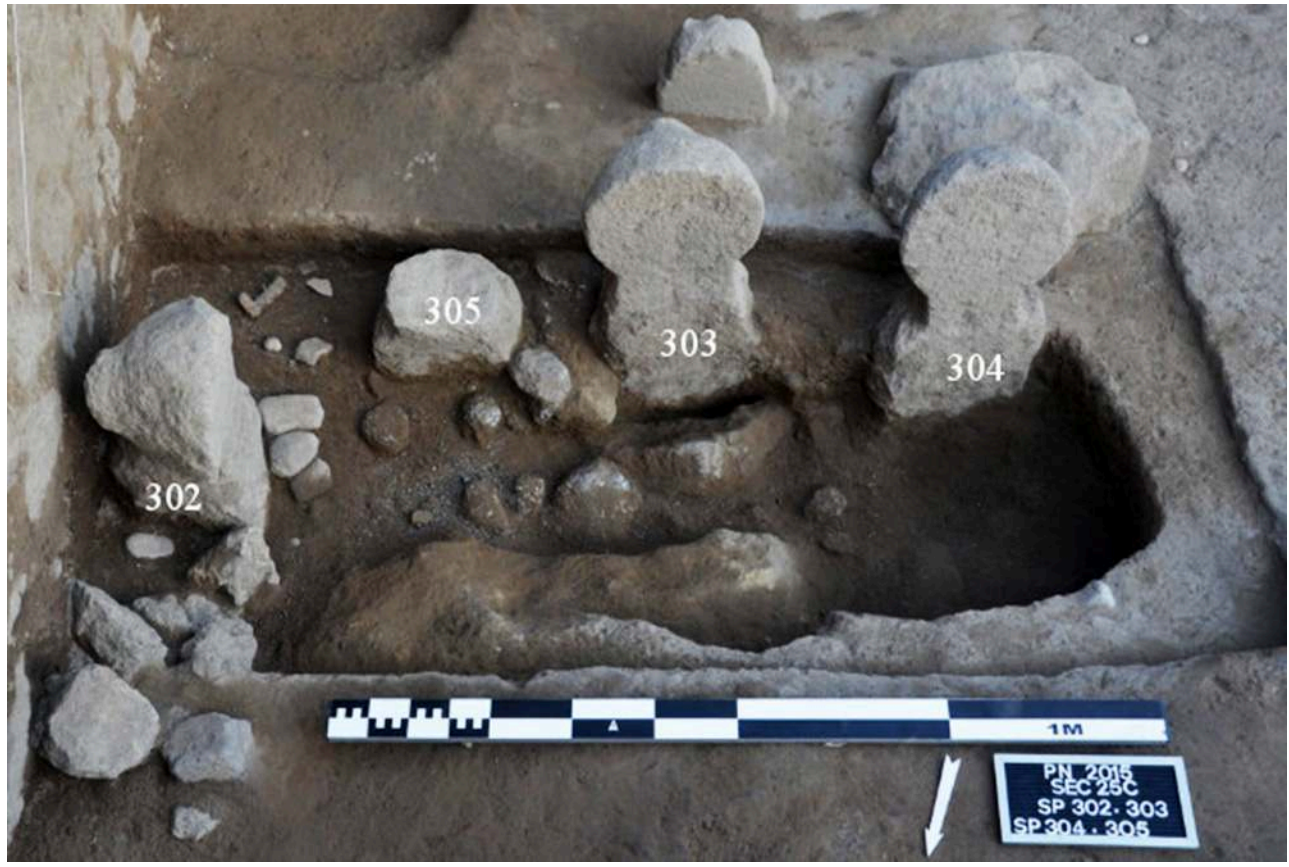

(c) Mission archéologique de Porta Nocera 2.

$\mathrm{Au}$ sein de l'ensemble, seules deux urnes en terre cuite ont été retrouvées. Elles contenaient des restes osseux qui n'ont pas encore été étudiés (fig. 6).

Fig. 6 - Détail du contenu de l'urne dans l'une des tombes de la concession 25c.

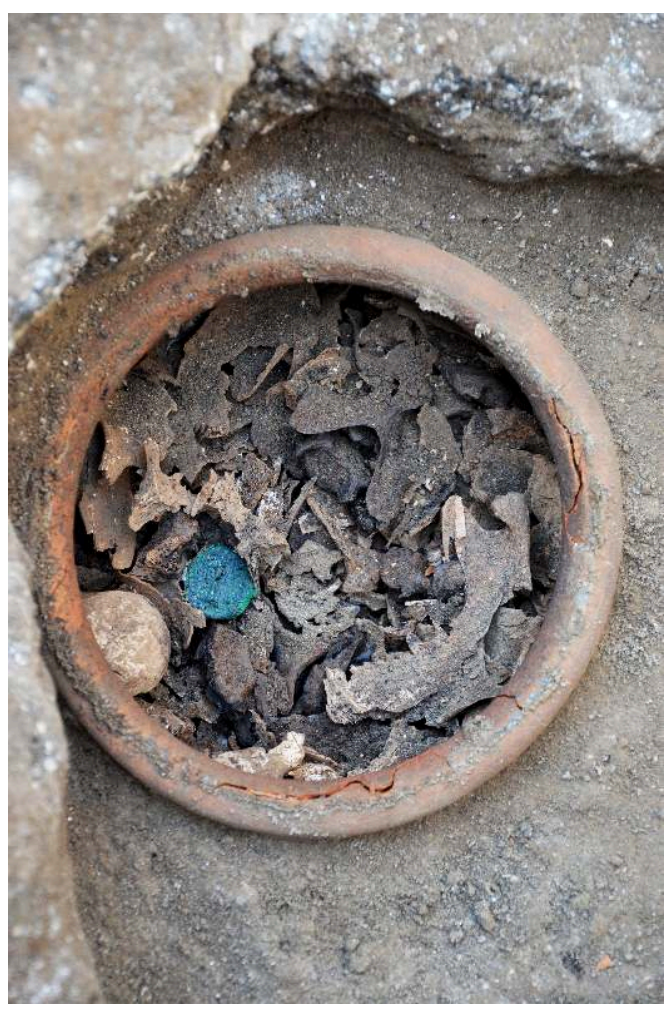

(c) Mission archéologique de Porta Nocera 2. 
Quelques dépôts - balsamaire en terre cuite, monnaies - sont associés à ces tombes. Il est difficile de comprendre pourquoi les autres fosses creusées dans le substrat n'ont pas reçu de dépôt alors que leur présence est marquée par des stèles en lave. Dans ce même secteur, une sépulture dépourvue de marquage au sol a été identifiée. Plus récente que les autres, elle se distingue par l'absence d'urne : les os ont été directement déposés dans une fosse quadrangulaire. Elle se situe à proximité d'une pierre au sommet arrondi qui pourrait correspondre à une borne délimitant l'emprise de la concession funéraire.

10 Enfin, une partie d'un bûcher funéraire a également été repéré plus au sud. Il se développe sous la niche funéraire de Castricia Prisca (25a) et n'a pu être que très partiellement fouillé. Une partie des os brûlés a pu être prélevée, ce qui permet d'espérer pouvoir mettre en évidence d'éventuels collages avec les tombes environnantes.

11 L'ensemble des monuments funéraires comme des tombes de la zone A n'ont pas encore été intégralement fouillés, mais les premiers résultats témoignent déjà d'une très forte disparité des pratiques, aussi bien pour la forme des monuments que pour les aménagements des tombes.

La fouille des niveaux situés entre les différents ensembles funéraires a permis de mieux comprendre l'évolution du secteur, même si l'ensemble n'a pas été totalement dégagé. Il apparaît que les trois ensembles étudiés cette année sont abandonnés selon des modalités différentes. Tous sont implantés dans un secteur ayant connu d'importants apports de colluvions. Le grand mausolée 27 OS est encore présent dans le paysage au moment de l'éruption de 79 apr. J.-C., mais il est partiellement ennoyé et aucune trace de fréquentation funéraire n'a pu être mise en évidence. Le monument 26a a quant à lui totalement disparu, une probable friche occupant cet espace. Quant à la concession 25c, elle s'est retrouvée noyée sous une épaisse couche de colluvions naturelles au sommet de laquelle la niche funéraire de Castricia Prisca et les tombes associées sont aménagées, probablement aux environs de 60 de n.è. Le caractère très modeste de cette construction comme la disparition de l'ancien enclos 26a donnent l'impression d'un terrain quelque peu délaissé, voire en partie abandonné. Le secteur qui formait comme une dent creuse au sein de la nécropole n'était manifestement pas concerné par la pression foncière. La forte épaisseur des niveaux de colluvions pourrait expliquer cet état de fait: cette partie de la nécropole de Porta Nocera était très certainement sujette à de fréquentes inondations.

13 L'étude de la voie n'a pas pu être achevée et ses liens avec les monuments funéraires demandent à être précisés. Il apparaît toutefois que cette voie a connu plusieurs recharges et que la chaussée a été plus ou moins large selon les états. Par ailleurs, un important niveau de colluvions naturelles sépare deux états de la voie, ce qui semble confirmer que ce secteur subissait fréquemment un apport massif de sédiments charriés par les eaux. Enfin, cette voie a longtemps surplombé les monuments voisins et elle était bordée d'un talus le long duquel ruisselaient de nombreux éléments retrouvés prisonniers dans les niveaux qui se sont progressivement amassés tout autour des monuments funéraires.

14 Il faut enfin signaler que la fouille des niveaux situés entre les enclos $25 \mathrm{c}$ et $26 \mathrm{a}$ a invité à rejeter l'une des hypothèses formulées l'an passé, à savoir la présence d'un bûcher dans ce secteur. 


\section{La Zone B (Porta Nocera Est). Le long de la route de Nocera : fouille de l'ensemble D/E/F nord et sud (voie et espaces funéraires)}

15 L'ouverture d'un nouveau secteur le long de la route de Nocera, le secteur B, était motivée par la volonté de comparer deux situations funéraires, l'une profondément marquée par la proximité des portes de la ville et des tombeaux de l'aristocratie locale, l'autre plus éloignée de l'espace urbain et en relation étroite avec une route fréquentée comme en témoignent les ornières profondes relevées sur le tronçon fouillé (fig. 7).

Fig. 7 - Photogrammétrie de la Zone B (le nord est en haut).

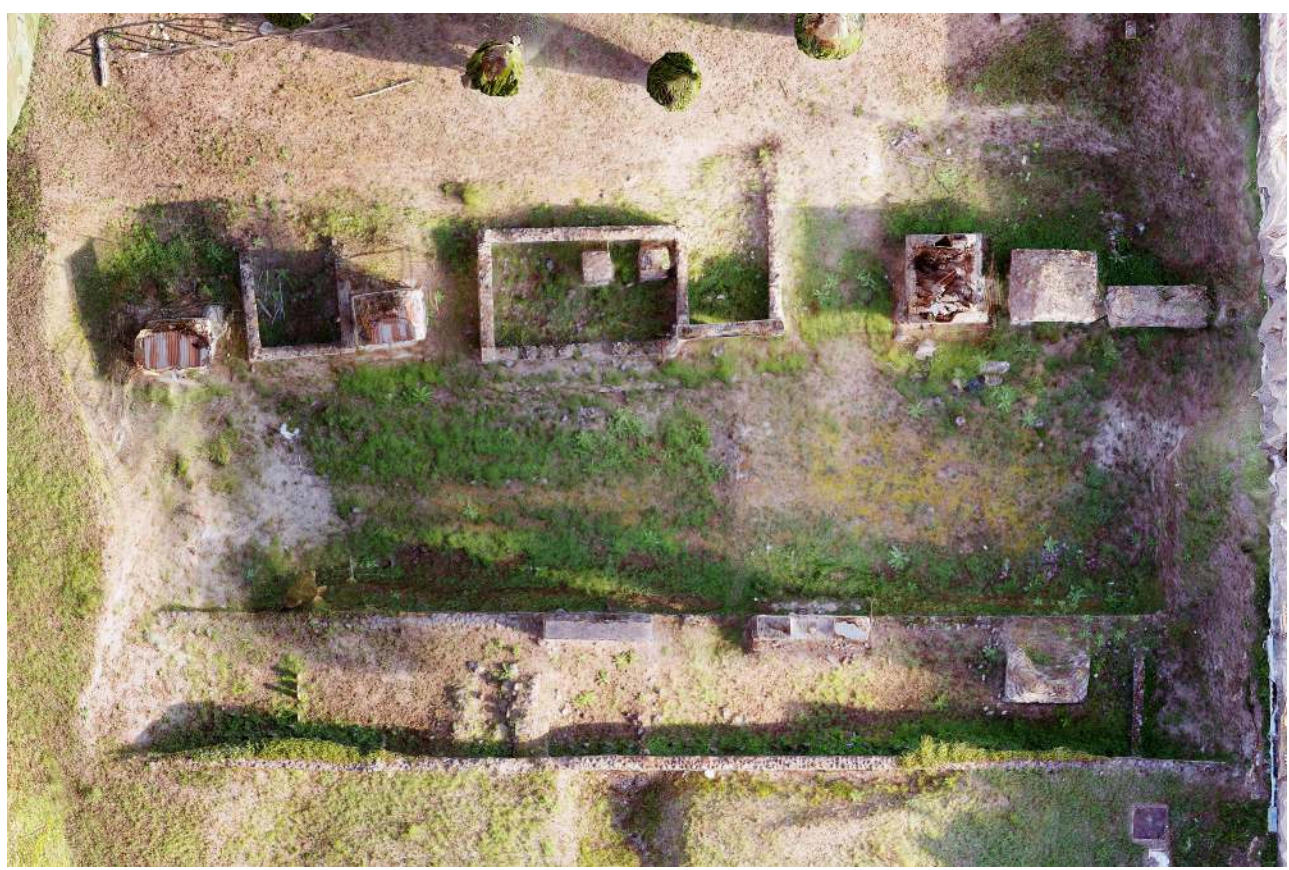




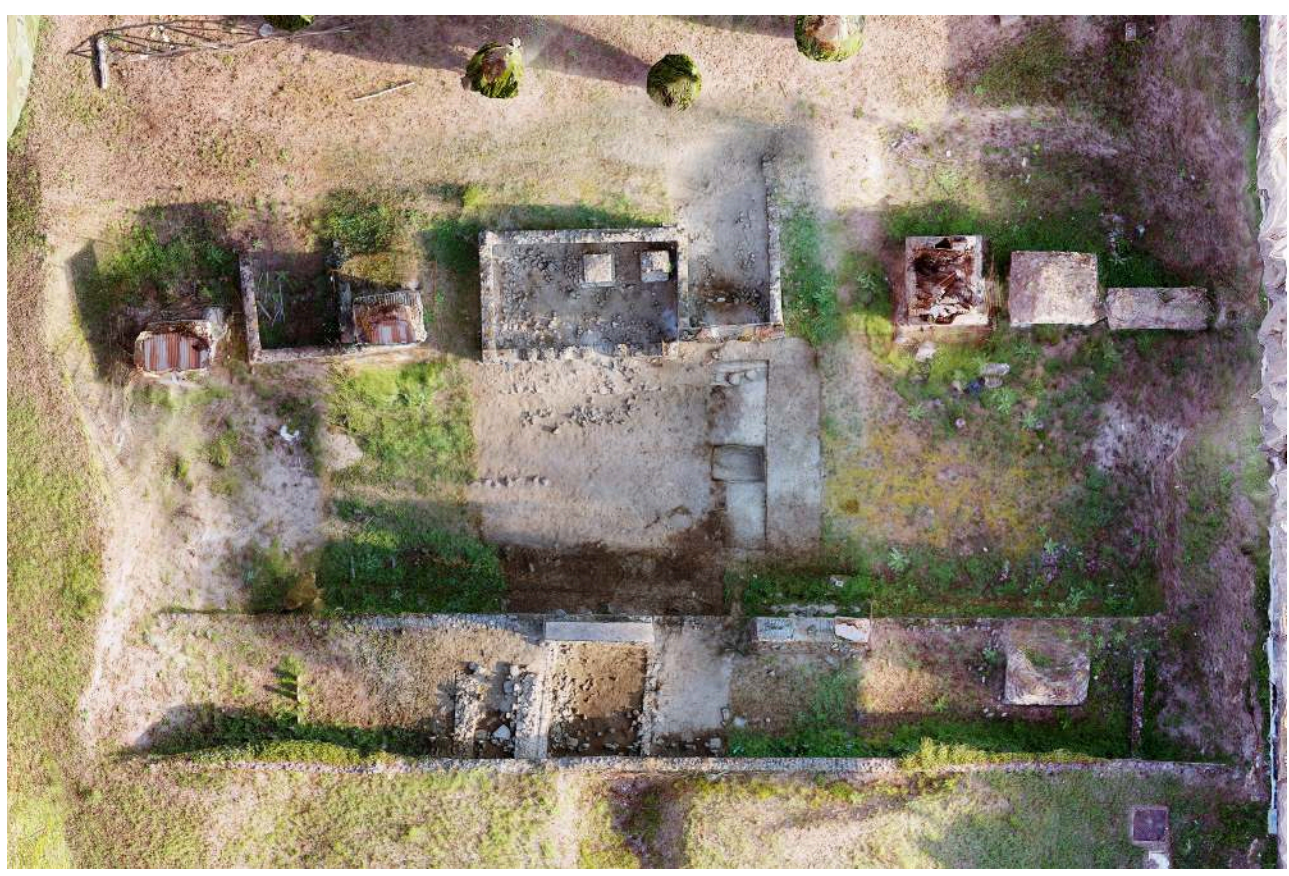

On distingue les monuments funéraires au nord et la terrasse funéraire au sud qui encadrent la route de Nocera au centre.

(c) Mission archéologique de Porta Nocera 2.

La question de la différence de statut social entre les morts enterrés aux portes de la ville et les morts installés plus loin, le long de la route, est évidemment posée; elle nécessite, pour trouver des réponses pertinentes, une approche très fine des vestiges et des tombes. Qu'est-ce qui détermine la différence de statut d'un défunt sinon le caractère imposant du monumentum, essentiel dans l'expression de la mémoire sociale ? L'aménagement de la tombe (définie par la mise en terre de l'amas osseux) peut-il renvoyer à des comportements sociaux différents? La gestion de l'espace observée dans le secteur B, notamment sur la terrasse sud, montre des différences avec le secteur A. Sont-elles pertinentes? L'entassement noté des morts, déposés parfois sans marquage de surface perceptible, par exemple dans l'enclos 3D où pas moins de 11 tombes sont aménagées dans $4 \mathrm{~m}^{2}$, tranche nettement avec l'aménagement rigoureux de l'espace des enclos du secteur A, occupés de façon à éviter les recoupements des sépultures. Comment interpréter de telles différences? Pourquoi les défunts de l'enclos 3D s'entassent-ils dans quelques mètres carrés, qui plus est à l'intérieur de murs bas très larges qui prennent autant de place que la concession elle-même? Dans l'enclos 23 OS fouillé près de la porte de Nocera, les tombes étaient installées par ensembles éloignés les uns des autres, ce qui les préservait des perturbations.

Dans ce secteur, l'une des questions posées est évidemment celle du recrutement funéraire, notamment pour les tombes alignées sur la terrasse, justifiant ainsi une fouille des sépultures les unes après les autres. Qui enterrait-on sur cette terrasse ? Les murs bas transversaux délimitent-ils des concessions funéraires? Le nombre de sépultures présentes permettra à terme de réfléchir à la question fondamentale, non élucidée encore, de la mort des immatures. Le premier projet (2003-2007) avait permis de montrer que les jeunes enfants étaient largement sous-représentés et que les périnatals étaient totalement absents dans les enclos familiaux proches des portes de la ville. Qu'en est-il un peu plus loin, sur la route de Nocera ? Sur la terrasse, une première série de 5 sépultures rapprochées (T7, T8, T9, T12 et T13) fouillées dans l'enclos 3E 
abrite au moins trois enfants, incinérés ou peut-être inhumé pour l'un d'eux. Il fait peu de doute que la poursuite de la fouille, par la multiplication des cas observés, nous permettra de cerner certaines des règles particulières qui encadraient la mort des immatures.

Dans le secteur B, le soin donné aux sépultures est révélateur de coutumes encadrant la mise au tombeau qu'il s'agira de découvrir et d'étudier pleinement dans les prochaines années. L'examen d'un nombre suffisant de tombes autorisant l'observation de logiques dans le recrutement funéraire selon les secteurs et les enclos prendra du temps, car, et la fouille de cette année est venue nous le rappeler, l'étude d'une tombe ou d'un groupe de tombes rapprochées prend beaucoup de temps, pouvant mobiliser une personne pendant toute la durée de la campagne. En effet, les sépultures ne sont pas seulement des cavités dans lesquelles sont déposées des urnes cinéraires. Il s'agit au contraire de constructions, souvent complexes et parfois destinées à être ré-ouvertes, dont il faut déceler les logiques et les partis pris jusqu'au dépôt des restes du défunt et, souvent, des résidus de crémation. Il s'agit aussi de dépôts secondaires, qui contiennent par conséquent les vestiges, souvent aussi nombreux que fugaces, mobiliers et osseux, de toute la séquence de transformation du mort, des funérailles jusqu'à la mise au tombeau. Les tombes sont enfin sujettes à des évolutions taphonomiques que l'archéologie permet de restituer. La tombe est-elle un locus religiosus, le domaine des dieux Mânes? Sans aucun doute, mais la sépulture du duumvir fouillée en 2015 (enclos 1F) est aussi un caisson fait de tuiles dans lequel furent déposés les restes incinérés de C. Veranius Rufus, duumvir de son état. Elle constitue tout autant le réceptacle des résidus de crémation dans lesquels furent retrouvés quelques artéfacts nous renseignant sur les funérailles. La tombe fut enfin soumise à une taphonomie active : non protégée, elle reçut pendant un certain laps de temps une eau de ruissellement abondante qui entraîna des désordres à l'intérieur du réceptacle, faisant flotter les os comme les charbons dans une alternance de dépôts sédimentaires retrouvés sur un mètre d'épaisseur et jonchés, à intervalle régulier, des ossements de crapauds piégés dans la tombe et tombés par le tube à libations.

En 2015, le travail effectué dans la zone B a concerné les enclos 1F et 1E, situés au nord de la route (F Nord et E Nord de D'Ambrosio - De Caro 1987), les enclos 3D et 3E (D Sud et $\mathrm{E}$ Sud de D'Ambrosio - De Caro 1987), situés sur la terrasse dominant la route au sud. Un sondage de 2 × 6,5 m a également été implanté sur la route. La fouille de l'enclos $1 \mathrm{~F}$ a concerné les niveaux récents d'éboulement et d'érosion, une partie des remblais d'une grande tranchée opérée en 1957-1958 et venue détruire l'arrière du monument ainsi que les niveaux d'occupation contemporains de l'implantation des huit sépultures identifiées de l'enclos. Deux tombes ont été fouillées, d'une part la sépulture $3 \mathrm{du}$ duumvir C. Veranius Rufus, constituée d'un caisson en tuile dans lequel fut versé, en vrac semble-t-il, l'ensemble du curage du bûcher avec les restes osseux du défunt, et d'autre part une sépulture d'immature, dépourvue de tout marquage et simplement constituée d'une fosse très peu profonde dans laquelle fut disposé un sac contenant les restes osseux. L'enclos mitoyen $1 \mathrm{E}$ est venu s'appuyer contre l'enclos $1 \mathrm{~F}$. Celui-ci englobe deux tombes aménagées dans un podium en maçonnerie (T1 appartenant à Novia Amoena et $\mathrm{T} 2$ à Cornelia Quieta), trois sépultures en pleine terre (T3 appartenant à $\mathrm{C}$. Novius Lupercus, $\mathrm{T} 5$ à $\mathrm{Cn}$. Turranius Primus et $\mathrm{T} 4$ à un individu anonyme) et une aire de crémation sur laquelle furent probablement brûlés les cinq défunts. La T1, constituée d'un caisson quadrangulaire en tuiles vidée lors des dégagements de 1983, a 
été nettoyée, révélant quelques esquilles osseuses. La fouille de la T5 a été initiée, mais arrêtée pour raison de sécurité, la fosse sépulcrale se prolongeant sous un mât d'échafaudage supportant une toiture mise en place à la suite des travaux de 1983. En revanche, le niveau d'occupation recouvrant l'ensemble de l'enclos a été fouillé, avec un prélèvement coordonné du matériel qu'il contenait (fig. 8). Concernant l'aire de crémation, située dans le tiers ouest de l'enclos, un protocole d'enregistrement particulier a été mis en place (corroyage de 75 carrés de $20 \mathrm{~cm}$ de côté, isolement du mobilier et tamisage du sédiment), susceptible de permettre la lecture de structures extrêmement fugaces et enchevêtrées.

Fig. 8 - Photogrammétrie de l'enclos $1 \mathrm{E}$ (le nord est en bas).

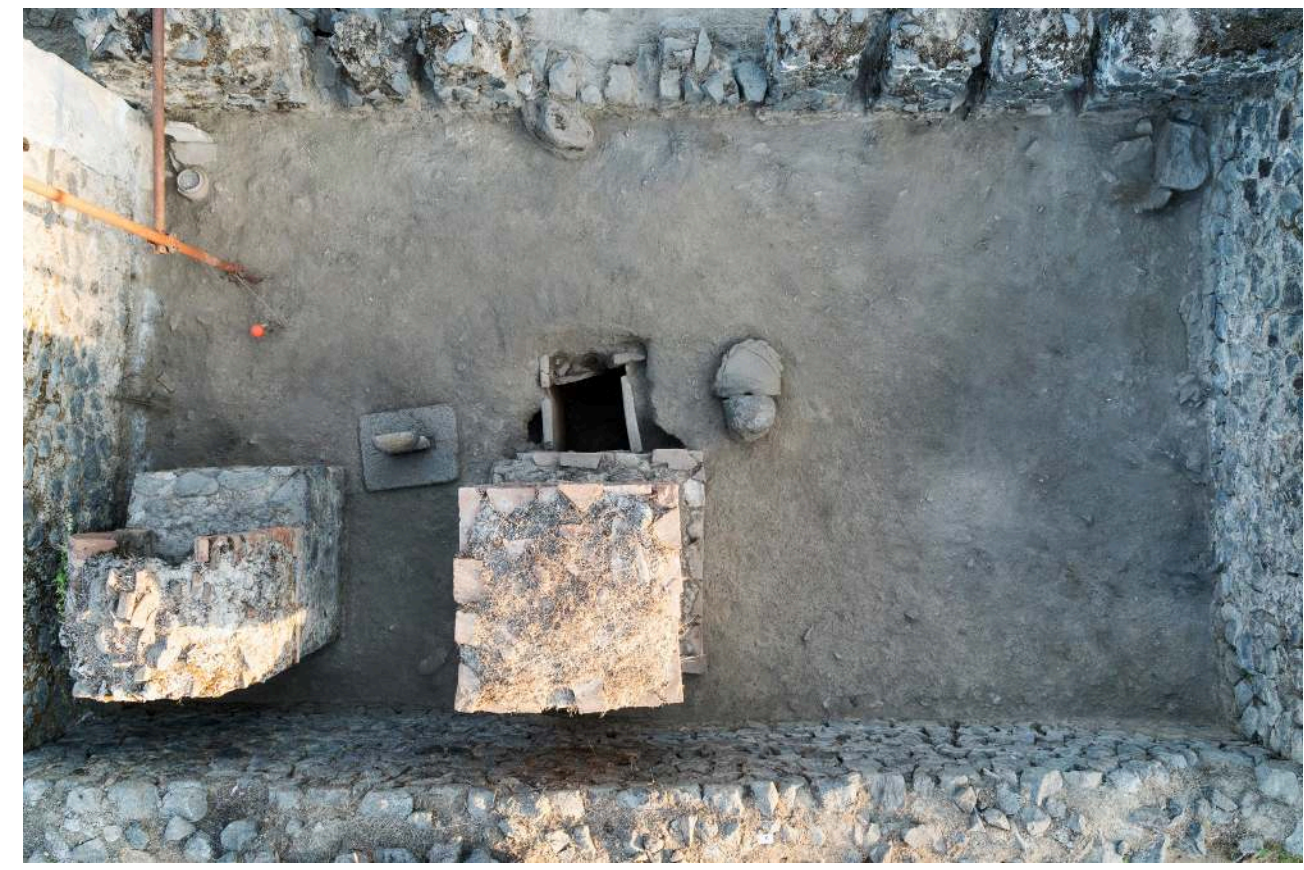

On distingue les tombes aménagées dans la partie orientale de l'enclos et l'aire de crémation organisée dans le tiers occidental.

(c) Mission archéologique de Porta Nocera 2.

Dans le prolongement de l'enclos $1 \mathrm{~F}$, un sondage a également concerné la route, encore recouverte d'une épaisse couche de pierres ponces. Sous le niveau déposé par l'éruption, la fouille a permis de dégager le dernier niveau de roulement de la chaussée. Il consiste en un sédiment limoneux cimenté par le tassement, comprenant des réparations liées à son entretien et des traces d'usures liées au passage des charrois. Les bas-côtés nord, composés de douze couches rapportées sur une épaisseur cumulée de $50 \mathrm{~cm}$, ont également été étudiés (fig. 9). 
Fig. 9 - Sondage implantée sur la route de Nocera.

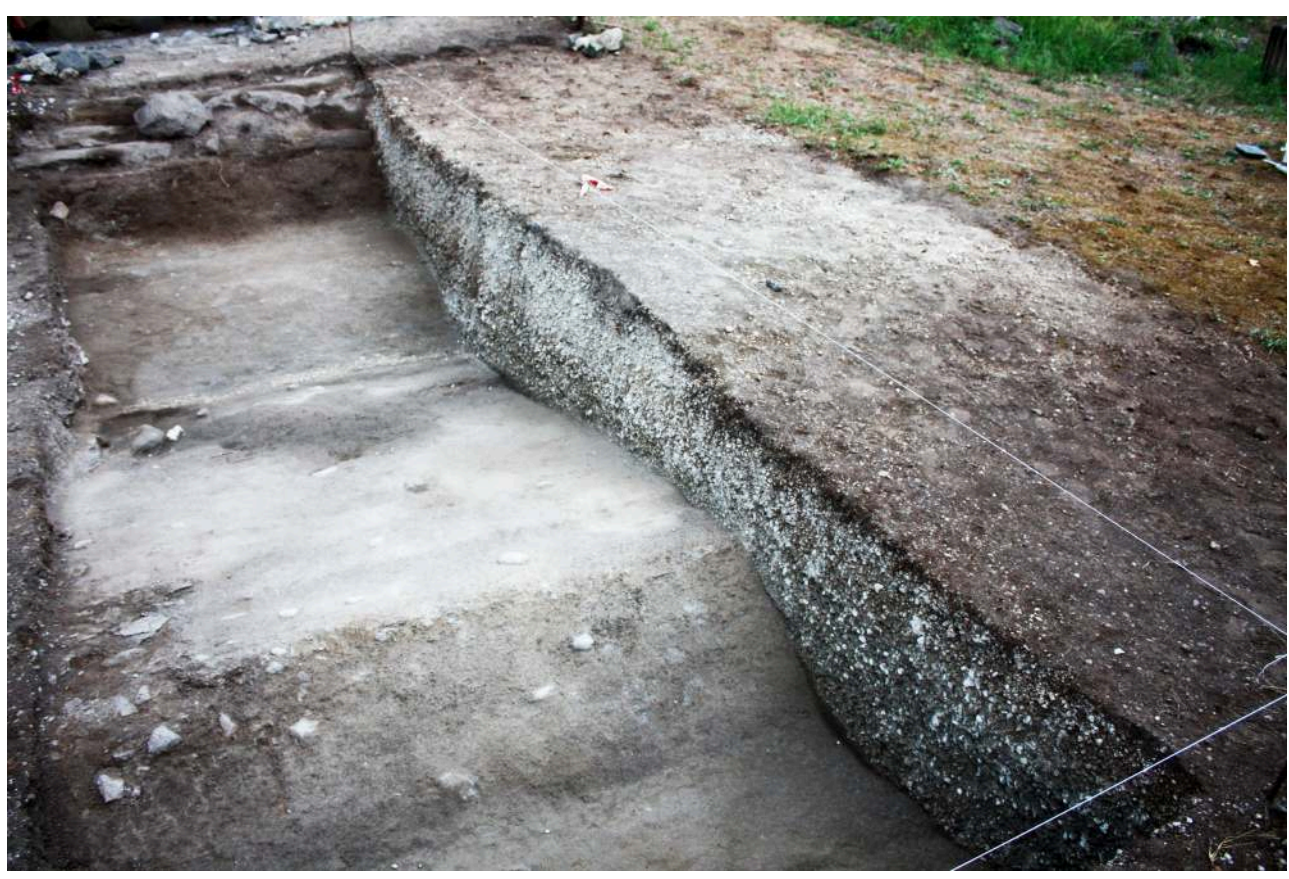

Au premier plan, la bande de roulement, au second, les aménagements du bas-côté permettant l'accès à l'enclos $1 F$.

(c) Mission archéologique de Porta Nocera 2.

20 Sur la terrasse sud qui surplombe la route, un premier travail a consisté à dégager les derniers niveaux d'occupation des enclos 3D et 3E. Dans la concession 3E, un premier ensemble de cinq tombes, aménagées le long du mur ouest, ont été fouillées (T7-9, T12 et T13), dont au moins trois appartiennent à des immatures (fig. 10). Trois sépultures disposées le long du mur sud ont également été fouillées (T3, T4 et T11), la tombe 11 présentant un aménagement particulier: simplement marquée à la surface par des tuiles disposées à plat, elle était constituée avec la panse d'une amphore Dr. 20 recouvrant le sac contenant l'amas osseux. L'enclos D n'a été que très partiellement fouillé. Deux sépultures ont été étudiées, la sépulture 9 et la sépulture 3 qui présentait un dispositif complexe visant sans aucun doute à protéger l'amas osseux grâce à l'emboîtement d'une urne, d'un contenant en plomb et d'une amphore renversée dont le pied assurait la fermeture de l'ensemble. 
Fig. 10 - Balsamaire en verre fragmenté découvert dans l'urne de la tombe 8 de l'enclos 3E.

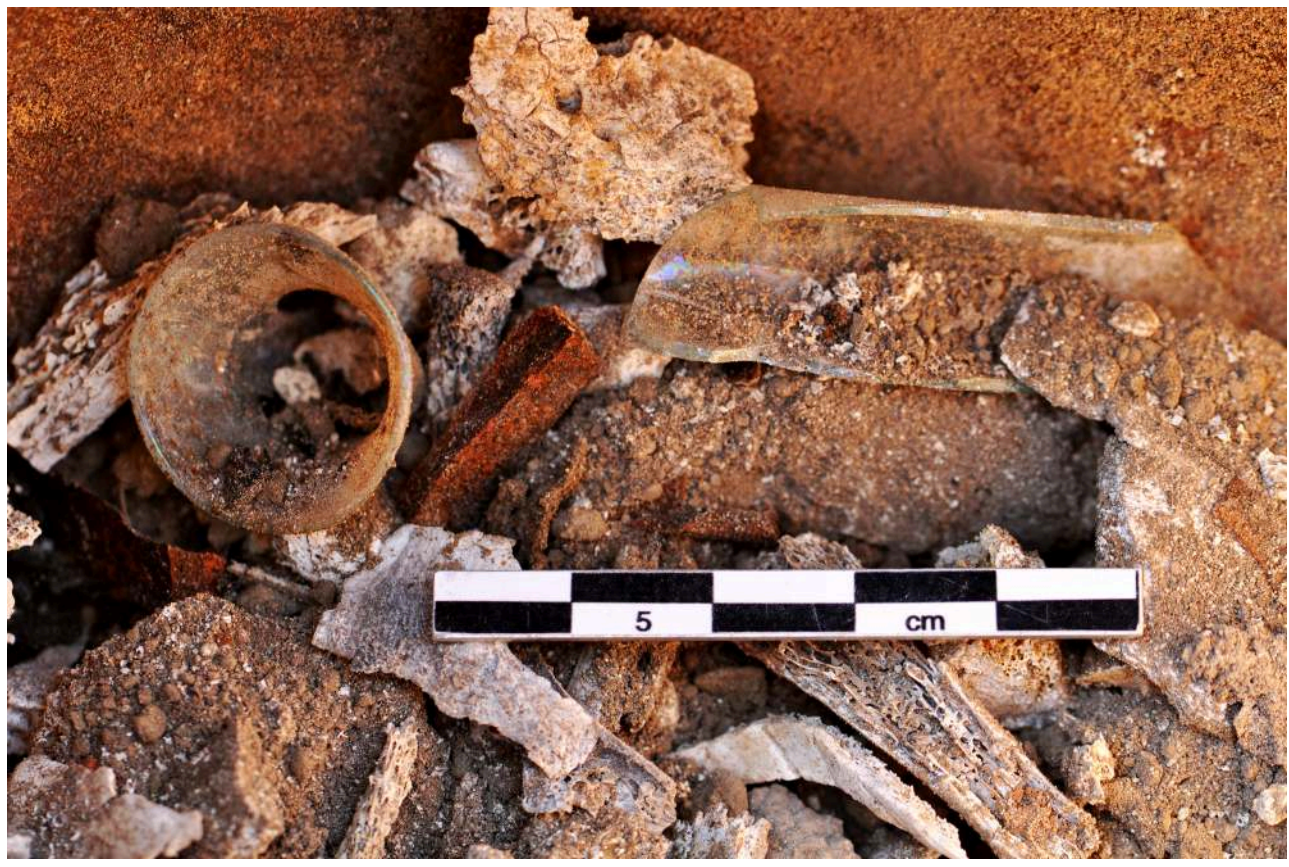

Il témoigne d'un geste opéré lors de la collecte des restes osseux du défunt.

(c) Mission archéologique de Porta Nocera 2.

21 Le travail sur deux secteurs différents nous permet également de réfléchir à la pédogénèse particulière des espaces funéraires. Selon nous, le terme de nécro-sol est pertinent dans le sens où l'activité funéraire, spécifique, est à l'origine d'une transformation des sols particulière qu'il s'agira de préciser dans les années à venir. Cette évolution des sols peut varier d'un secteur à l'autre comme nous avons pu le montrer cette année. Dans le secteur A, le principe essentiel est celui d'un exhaussement des sols funéraires, provoqué par les creusements successifs des tombes et l'activité funéraire, mais également par des apports de terrain anthropiques (lorsqu'il s'agit d'aménager une nouvelle concession) ou par des apports naturels parfois importants. Dans le secteur $B$, c'est le phénomène contraire que nous avons pu mettre en évidence : le caractère volatil, pulvérulent, du sédiment est à l'origine d'un phénomène surprenant de déflation des sols. La fréquentation des enceintes funéraires et les intempéries sont à l'origine d'une désagrégation des sols qui sont emportés par le vent ou les fortes pluies. Le résultat est une érosion des niveaux à l'intérieur des enclos et une «compression» stratigraphique du matériel. Ce phénomène sera observé plus précisément dans les années à venir. Dans une ville aussi étudiée que Pompéi, l'étude des transformations du sol sous l'action anthropique et naturelle est à notre sens un enjeu majeur de la compréhension du site et de son évolution. 


\section{BIBLIOGRAPHIE}

Creissen - Van Andringa - Duday 2015 = Th. Creissen, W. Van Andringa, H. Duday (dir.), La nécropole romaine de Porta Nocera à Pompéi : le secteur 26 OS, dans Chronique des activités archéologiques de l'École française de Rome [En ligne], 2015, mis en ligne le 04 mai 2015. URL : http://

cefr.revues.org/1352.

D’Ambrosio - De Caro 1984 = A. D’Ambrosio, S. De Caro, Un impegno per Pompei : fotopiano e documentazione della Necropoli di Porta Nocera, Milan, 1984.

D’Ambrosio - De Caro 1987 = A. D'Ambrosio, S. De Caro, La Necropoli di Porta Nocera. Campagna di Scavo 1983, dans P. Zanker et H. von Hesberg (dir.), Römische Gräberstrassen : Selbstdarstellung, Status, Standard: Kolloquium in München vom 28. bis 30. Oktober 1985, Munich, 1987, p. 199-228.

Duday 2009= H. Duday, The archaeology of the dead: lectures in archaeothanatology, Oxford-Oakville, 2009.

Duday - Van Andringa 2013 = H. Duday, W. Van Andringa, Des formes et du temps de la mémoire dans une nécropole de Pompéi, dans Les Nouvelles de l'Archéologie, 132, 2013, p. 48-54.

Duday - Van Andringa s.p. = H. Duday, W. Van Andringa, Archeology of Memory: about the forms and the time of memory in one necropolis of Pompeii, dans J. Knust, C. Moser (ed.), Rituals matter: the Materiality of Ancient Religions, Rome, s.p.

Van Andringa et al. $=\mathrm{W}$. Van Andringa, H. Duday, S. Lepetz et D. Joly, T. Lind et al., Mourir à Pompéi : fouille d'un quartier funéraire de la nécropole romaine de Porta Nocera (2003-2007), Rome, 2013 (Collection de l'École française de Rome, 468).

Van Andringa - Creissen - Duday 2014 = W. Van Andringa, Th. Creissen, H. Duday (dir.), Porta Nocera 2, Campagne de fouille 2014 : naissance et développement d'un paysage funéraire romain (I ${ }^{\text {er }}$ siècle av. J.-C. - Irr siècle apr. J.-C.), Pompéi, 2014, rapport de fouille.

Van Andringa - Creissen - Duday 2015 = W. Van Andringa, Th. Creissen, H. Duday (dir.), Porta Nocera 2, Campagne de fouille 2015 : naissance et développement d'un paysage funéraire romain (I ${ }^{\text {er }}$ siècle av. J.-C. - Irr siècle apr. J.-C.), Pompéi, 2015, rapport de fouille.

\section{NOTES}

1. La campagne 2015 du programme de recherche PORTA NOCERA 2 s'est déroulée du 24 août au 27 septembre 2015. Nous tenons à remercier particulièrement le Prof. Soprintendente Massimo Osanna, la Dott.ssa Annalisa Capurso, funzionario archeologo, et l'Assistente Vicenzo Sabini qui ont rendu possible la mise en œuvre de ce programme d'étude ainsi que Catherine Virlouvet, directrice de l'École française de Rome, Stéphane Bourdin, directeur des études pour l'Antiquité, sans oublier Giulia Cirenei, assistante scientifique pour l'Antiquité. Notre profonde gratitude va également aux institutions qui portent le programme, l'École française de Rome, l'Université de Lille 3 et le laboratoire Halma ainsi qu'Éveha international et le Prix Clio.

2. D’Ambrosio - De Caro 1987. Un rapport d'opération a été déposé auprès des différentes institutions partenaires : Van Andringa W., Creissen Th., Duday H. (dir.) et al., Porta Nocera 2 Campagne 2015. Naissance et développement d'un paysage funéraire romain ( ${ }^{\text {er }}$ siècle av. J.-C .- I ${ }^{\text {er }}$ siècle apr. J.-C.), Rapport Final d'Opération, Pompéi, 2015. Le programme de recherches est présenté sur 
notre site Web: http://www.deathinpompeii.com ainsi que sur le site d'Éveha International: http://eveha-international.com/fr/intervention/pompeii-porta-nocera.

\section{INDEX}

institutions Programme de recherche de l'École française de Rome en collaboration avec l'université de Lille 3 (Centre de recherches Halma), la société archéologique Éveha International et la Soprintendenza archeologica di Pompei, avec la participation du laboratoire d'anthropologie de Bordeaux et la société Archeodunum.

Index géographique : Pompéi, Porta Nocera

\section{AUTEURS}

\section{WILLIAM VAN ANDRINGA}

Université Lille 3, Helsinki Collegium for Advanced Study - william.va[at]free.fr

\section{THOMAS CREISSEN}

Éveha International, Université de Tours - thomas.creissen[at]eveha.fr

\section{HENRI DUDAY}

CNRS, Université de Bordeaux - h.duday[at]anthropologie.u-bordeaux1.fr 